\title{
NMR metabolomics of symbioses between bacterial vaginosis associated bacteria
}

Victoria Horrocks ${ }^{1}$, Charlotte K. Hind ${ }^{2}$, Matthew E. Wand ${ }^{2}$, Joel Chan ${ }^{1}$, Jade C. Hopkins ${ }^{1}$, Georgina L. Houston ${ }^{1}$, Rachel M. Tribe ${ }^{3}$, J. Mark Sutton ${ }^{1,2}$, A. James Mason ${ }^{1 *}$

${ }^{1}$ Institute of Pharmaceutical Science, School of Cancer \& Pharmaceutical Science, King's College London, Franklin-Wilkins Building, 150 Stamford Street, London, SE1 9NH, United Kingdom

${ }^{2}$ Technology Development Group, Research and Evaluation Division, UK Health Security Agency, Porton Down Salisbury, UK ${ }^{3}$ Department of Women and Children's Health, School of Life Course Sciences, Faculty of Life Sciences and Medicine, King's College London,

Keywords: bacterial vaginosis, spontaneous preterm birth, vaginal microbiome, Prevotella bivia, Gardnerella vaginalis, Peptostreptococcus anaerobius, Atopobium vaginae, Mobiluncus curtisii.

ABSTRACT: Bacterial vaginosis (BV) is a dysbiosis of the vaginal microbiome, characterised by low levels of lactobacilli and overgrowth of a diverse group of bacteria, and associated with higher risk of a variety of infections, surgical complications, cancer and spontaneous preterm birth (PTB). Despite the lack of a consistently applicable aetiology, Prevotella spp. are often associated with both BV and PTB and P. bivia has known symbiotic relationships with both Peptostreptococcus anaerobius and Gardnerella vaginalis. Higher risk of PTB can also be predicted by a composite of metabolites linked to bacterial metabolism but their specific bacterial source remains poorly understood. Here we characterise diversity of metabolic strategies among BV associated bacteria and lactobacilli and the symbiotic metabolic relationships between $P$. bivia and its partners and show how these influence the availability of metabolites associated with BV/PTB and/or pro- or anti-inflammatory immune responses. We confirm a commensal relationship between $P$ e. anaerobius and $P$. bivia, refining its mechanism; $P$. bivia supplies tyrosine, phenylalanine, methionine, uracil and proline, the last of which leads to a substantial increase in overall acetate production. In contrast, our data indicate the relationship between P. bivia and G. vaginalis strains, with sequence variant G2, is mutualistic with outcome dependent on the metabolic strategy of the G. vaginalis strain. Seven $G$. vaginalis strains could be separated according to whether they performed mixed acid fermentation (MAF) or bifid shunt (BS). In co-culture, P. bivia supplies all $G$. vaginalis strains with uracil and received substantial amounts of asparagine in return. Acetate production, which is lower in BS strains, then matched that of MAF strains while production of aspartate increased for the latter. Taken together, our data show how knowledge of inter- and intraspecies metabolic diversity and the effects of symbiosis may refine our understanding of the mechanism and approach to risk prediction in BV and/or PTB.

\section{Introduction}

Bacterial vaginosis (BV) is regarded as a disruption of the lower genital tract microbiota with a shift from lactobacilli dominance to include a greater proportion of a range of species including members of the genera Gardnerella, Prevotella, Atopobium, Mobiluncus, and Peptostreptococcus as well as Sneathia, Leptotrichia, Mycoplasma, and BV-associated bacterium 1 (BVAB1) to BVAB3. ${ }^{1}$ Despite the lack of consistent aetiology documented in women with BV, vaginal dysbiosis involving a plethora of species, irrespective of whether symptoms of BV are present, promotes local inflammation and is associated with a wide array of health problems. ${ }^{1}$

A specific complication that may be related to $B V$ is a 2-fold increased risk of spontaneous preterm birth (PTB). ${ }^{2,3}$ However, screening for asymptomatic BV in pregnancy in low-risk groups has not aided preterm birth prediction 

available under aCC-BY 4.0 International license.

have pursued the association between the vaginal microbiome and PTB risk, ${ }^{5-16}$, including our own, ${ }^{15}$ and many of the species identified as associated with higher risk of PTB overlap with those associated with BV.

Changes in microbiota composition are reflected in variations in bacterial derived metabolite profiles, ${ }^{11,15,17}$ which may have functional impact. ${ }^{18-21}$ Consistent with the microbiome studies, elevated vaginal lactate, which is the major product of the lactobacilli, and succinate have been found to be associated with term delivery, ${ }^{11}$ while elevated acetate was subsequently found to be higher in women who delivered preterm compared with term. ${ }^{17}$ A role for these metabolites in BV has also been considered, ${ }^{18,21}$ with two studies agreeing that low lactate and high acetate and propionate are characteristic of BV. ${ }^{22,23}$ Recently, we have shown that combining microbiome and metabolome into composite models has predictive value for preterm birth. ${ }^{15} \mathrm{~A}$ composite of metabolites which include lactate and acetate but also, aspartate, leucine, tyrosine and betaine associated with risk of PTB < 37 weeks while risk of PTB $<34$ weeks was identified by a composite comprising L. crispatus, L. acidophilus, glucose and, again, aspartate.

Although multiple studies have identified Prevotella spp. as being associated with both BV, and preterm birth, ${ }^{9,12,13,15}$ their presence has not been found to be predictive of PTB. ${ }^{15}$ However, their residence within the vagina correlates with that of a number of other bacteria including Gardnerella vaginalis, ${ }^{15,16}$ and $P$. bivia is known to enjoy symbiotic interactions with both Peptostreptococcus anaerobius and G. vaginalis. ${ }^{24-26}$ Two groups have found an association between preterm birth and G. vaginalis, ${ }^{7,9,16}$ but its presence alone does not predict PTB. There is though, reason to consider whether the substantial diversity of $G$. vaginalis affects the ability to establish its functional role(s) in both BV and preterm birth. ${ }^{27}$ Studies of microbial communities often sequence and quantify specific marker genes and cluster such sequences into Operational Taxonomic Units (OTUs). Although such OTUs have been generally shown to have high levels of ecological consistency, ${ }^{28}$ and the approach remains popular and useful, there remains the possibility that functionally relevant differences in bacterial behaviour are obscured by this approach. Indeed, in one study that confirmed an association between $G$. vaginalis and preterm birth, high-resolution statistical bioinformatics was used to detect nine unique $G$. vaginalis $16 \mathrm{~S}$ rRNA sequence variants and this revealed that only one of three $G$. vaginalis clades was responsible for the association of the

In addition, the role of the otherwise dominant lactobacilli may also be critical in defining PTB risk, with Lactobacillus crispatus dominance frequently associated with term delivery. ${ }^{9,10,13,15,16}$ The picture for $L$. iners is less clear. One study showed an association with PTB, ${ }^{10}$ but two subsequent studies found none.,${ }^{9,15}$ Instead they found frequent co-existence of $L$. iners with $G$. vaginalis, ${ }^{9}$ which contrasts with $L$. crispatus where an exclusionary relationship with $G$. vaginalis is found ${ }^{9,16}$ or positive correlation with $\mathrm{BV}$ associated bacteria including $P$. bivia. ${ }^{15}$

Given the valuable utility of the NMR metabolomics approach for identifying risks associated with vaginal dysbiosis and predicting PTB, and associations with differing microbiome states likely to have functional impact, there is an unmet need to understand bacterial contributions to the vaginal metabolome in more detail. To this end, we aimed to establish a mechanistic basis for a mutualistic symbiotic relationship between $P$. bivia and $G$. vaginalis and contrast this with the commensal relationship between $P$. bivia and Pe. anaerobius. We characterise the diverse metabolic strategies of a panel of $G$. vaginalis isolates and determine how this influences symbiosis with $P$. bivia. In addition, we compare metabolism across a panel of lactobacilli to highlight that variation in metabolic strategy is not limited to BV/sPTB associated bacteria and that the metabolite background will likely vary according to microbiome community state type (CST). ${ }^{5}$ The information provided by the present study suggests ways of refining prediction models that include metabolite data and gives insight into how bacterial metabolism and symbiosis influence each other, with implications for functional impact and clinical outcomes. 


\section{Experimental procedures}

Isolates. Gardnerella vaginalis 11292, 10915 and 10287, Peptostreptococcus anaerobius 11460, Prevotella bivia 11156, Atopobium vaginae 13935 and Mobiluncus curtisii 11656 were obtained from the National Collection of Type Cultures (NCTC). All other bacteria were isolated from swabs collected from pregnant women recruited with informed written consent via the INSIGHT study (NHS Human Research Authority, London - City and East Research Ethics Committee 13LO/1393) or from Salisbury District Hospital (SDH) microbiology lab. Samples from SDH were received from the microbiology laboratory following diagnostic testing. All identifiers were re-moved by the diagnostic laboratory'. The swabs were maintained at ambient temperature during transport in liquid amies buffer and were used immediately or frozen at $-80^{\circ} \mathrm{C}$ until use. $100 \mu \mathrm{l}$ of the buffer solution was either plated onto tryptic soy agar (TSA) and De Man, Rogosa and Sharpe (MRS) agar plates and incubated at $37^{\circ} \mathrm{C}$ for 48 hours under aerobic condition or plated onto TSA, MRS agar and Columbia blood agar (CBA), containing 5\% defibrinated sheep's blood (Oxoid), and incubated at $37^{\circ} \mathrm{C}$ for 48 hours under anaerobic conditions as outlined below. Single colonies were streaked to purity and identified using MALDI-TOF spectrometry (MALDI Biotyper ${ }^{\circledR}$, Bruker Daltonics GmbH \& Co. KG, DE).

Bacterial culture. All G. vaginalis isolates, Pe. anaerobius, P. bivia, M. curtisii and A. vaginae were plated onto CBA (Oxoid, Hampshire, UK) containing $5 \%$ defibrinated sheep's blood (Oxoid) and incubated at $37^{\circ} \mathrm{C}$ for 48 hours under anaerobic conditions generated using Thermo Scientific ${ }^{\mathrm{TM}}$ Oxoid $^{\mathrm{TM}}$ AnaeroGen $^{\mathrm{TM}}$. L. iners was plated under the same conditions for 72 hours. All other Lactobacillus species were plated onto MRS agar (Sigma Aldrich) and incubated at $37^{\circ} \mathrm{C}$ for 48 hours under anaerobic conditions. For initial overnight cultures a $1 \mu \mathrm{l}$ loop of culture was used to inoculate $5 \mathrm{ml}$ of brain-heart infusion (BHI) media with $5 \%$ horse serum and incubated at $37^{\circ} \mathrm{C}$ for 48 hours under anaerobic conditions without shaking. For monoculture samples, $50 \mu$ l of overnight culture was added to 5 $\mathrm{ml}$ of fresh $\mathrm{BHI}$ with $5 \%$ horse serum and incubated at $37^{\circ} \mathrm{C}$ for 48 hours under anaerobic conditions without shaking. For coculture of $P$. bivia with $G$. vaginalis or Pe. anaerobius, from overnight cultures, a 1:1 mix of each species was used to inoculate $5 \mathrm{ml}$ of fresh $\mathrm{BHI}$ with $5 \%$ horse serum and incubated at $37^{\circ} \mathrm{C}$ for 48 hours under anaerobic conditions without shaking.

MIC testing. The minimum inhibitory concentrations (MICs) were measured using a broth microdilution method in polypropylene plates (Greiner). From an overnight culture in $\mathrm{BHI} 100 \mu \mathrm{l}$ of bacterial culture totalling an $\mathrm{OD}_{600} \mathrm{Of}$ 0.1 was added to $100 \mu \mathrm{l}$ of $\mathrm{BHI}$ media containing antibiotic. After 48 hours of incubation at $37^{\circ} \mathrm{C}$ under anaerobic conditions the optical density at a wavelength of $600 \mathrm{~nm}$ was read. The lowest concentration of antibiotic where there was no growth $\left(\mathrm{OD}_{600}<0.1\right)$ determined the MIC.

NMR metabolomics. For preparation of samples to be used in metabolomics bacterial cultures were pelleted by centrifuge at $5000 \mathrm{rpm}$ at $4^{\circ} \mathrm{C}$. Supernatant was filtered with $0.22 \mu \mathrm{m}$ membrane to remove any bacterial cells and large debris and were stored at $-80^{\circ} \mathrm{C}$ until use. To aid suppression of the water signal and deuterium lock and act as an internal reference, $60 \mu \mathrm{l}$ of $\mathrm{D}_{2} \mathrm{O}+3$-(trimethylsilyl)propionic-2,2,3,3-d4 acid sodium salt (TSP-d4) was added to $570 \mu \mathrm{l}$ of filtered supernatant. The $\mathrm{pH}$ of all samples was adjusted using $\mathrm{NaOH}$ to within $0.2 \mathrm{pH}$ units of the $\mathrm{BHI}$ media control. ${ }^{1} \mathrm{H}$ NMR spectra were recorded on Bruker $600 \mathrm{MHz}$ Bruker Avance III NMR spectrometer (Bruker BioSpin, Coventry, United Kingdom) equipped with a $5 \mathrm{~mm}{ }^{1} \mathrm{H},{ }^{13} \mathrm{C},{ }^{15} \mathrm{~N} \mathrm{TCl}$ Prodigy Probe and a cooled sample changer with all samples kept at $4{ }^{\circ} \mathrm{C}$. The $1 \mathrm{D}$ spectra were acquired under automation at a temperature of $298 \mathrm{~K}$ using Carr-Purcell-Meiboom-Gill presaturation (CMPG) pulse sequence (cpmgrp1). The parameters of spectra acquisition are 32 transients, a spectral width of 20.83 ppm and 65,536 datapoints. For assignment of metabolite peaks additional spectra, Total correlation spectroscopy (TOCSY), ${ }^{1} \mathrm{H}^{-13} \mathrm{C}$ heteronuclear single quantum correlation spectroscopy and J-resolved spectroscopy (JRES), were acquired from a pooled sample containing a small volume of all samples. Resonance positions are quoted in ppm with respect to the methyl peak of TSP-d4 at $0.0 \mathrm{ppm}$. 

available under aCC-BY 4.0 International license.

126 All spectra were Fourier transformed in Bruker software and adjusted using automatic baseline correction and 127 phasing in Bruker TopSpin 4.1.3. Multiple databases were used for the assignment of metabolites; Chenomx NMR 128 suite software (Chenomx Inc, Canada), Human Metabolome Database (HMDB) and Biological Magnetic Resonance 129 Data Bank (BMRB). ${ }^{29}$ To convert NMR intensity to $\mathrm{mM}$ concentration the Chenomx software programme was used 130 calibrated to the concentration of TSP-d4 present in the sample. For multivariate analysis the intensity of all sam131 ples was normalised using probabilistic quotient normalisation (PQN). ${ }^{30}$ For visualisation of data, python packages 132 numpy, matplotlib, seaborn, pandas and scipy were used.

133 Sequencing. All isolates identified as G. vaginalis from MALDI-TOF were also confirmed through whole genome 134 sequencing. DNA was extracted from overnight culture in $\mathrm{BHI}$ using the GenElute ${ }^{\mathrm{TM}}$ Bacterial Genomic DNA Kits 135 (Sigma Aldrich). DNA was tagged and multiplexed with the Nextera XT DNA kit (Illumina, San Diego, US) and se136 quenced by Public Health England Genomic Services and Development Unit, (PHE-GSDU) on an Illumina (HiSeq 137 2500) with paired-end read lengths of $150 \mathrm{bp}$. A minimum $150 \mathrm{Mb}$ of Q30 quality data were obtained for each 138 isolate. FastQ files were quality trimmed using Trimmomatic ${ }^{31}$. SPAdes 3.1.1 was used to produce draft chromo139 somal assemblies, and contigs of less than $1 \mathrm{~kb}$ were filtered out ${ }^{32}$. Whole genome alignment and phylogenetic 140 tree generation were performed using progressive alignment in Mauve Version 20150226 build 10. Tree visualisa141 tion was performed in FigTree Version 1.4.3.
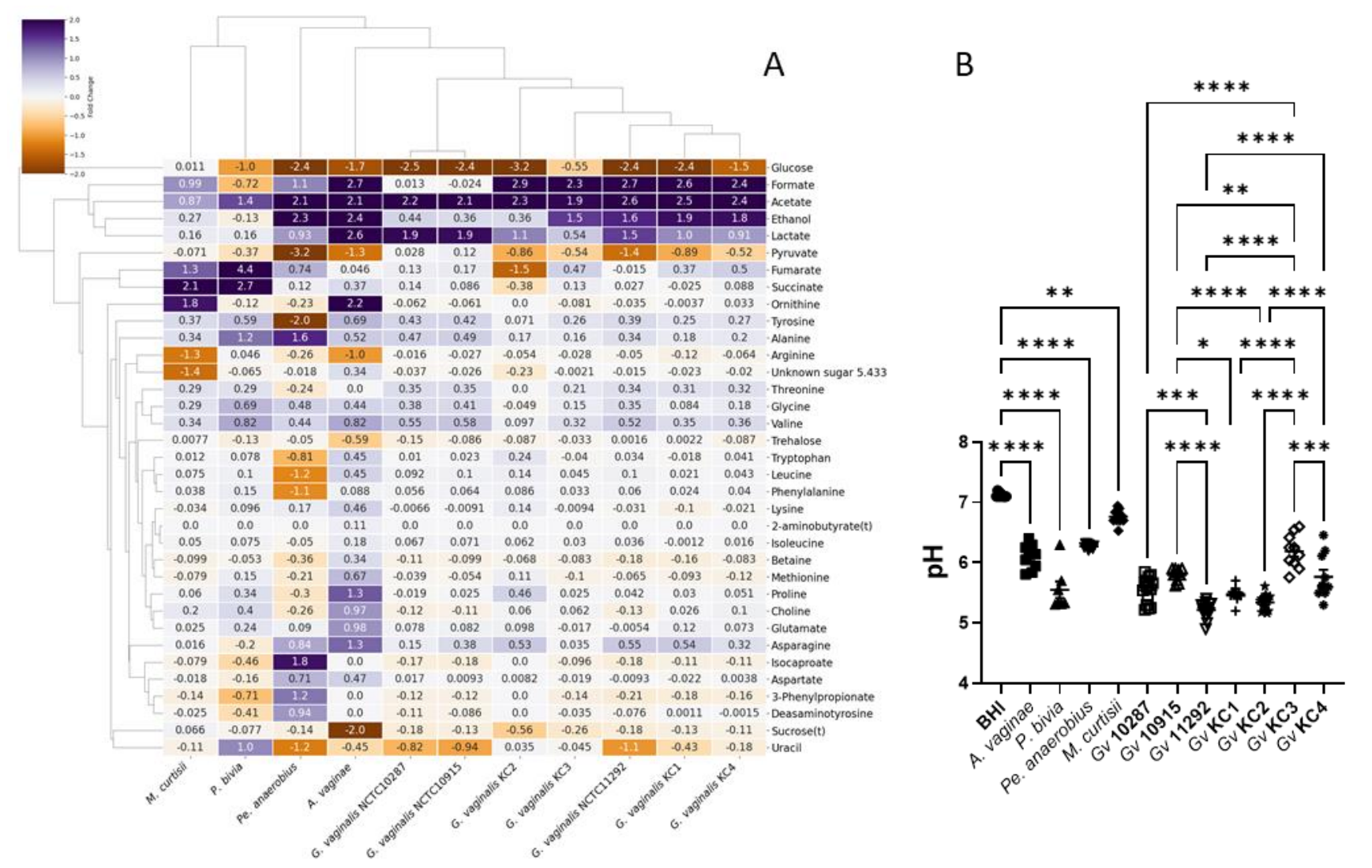

Figure 1. Diversity of bacterial vaginosis associated bacteria metabolism, when cultured in brain heart infusion. The heatmap (A) compares the metabolite fold change from ${ }^{1} \mathrm{H}$ NMR of spent culture media and enables the major metabolites produced by each isolate and the key differences in metabolic strategy to be revealed. The resulting acidification of the spent culture media accordingly varies (B). Comparisons are shown between fresh $\mathrm{BH}$ and the five non-G. vaginalis conditions and between each of the $G$. vaginalis strains, as determined by One-way ANOVA with Tukey correction for multiple comparisons. 
150 To better understand the contribution of different bacteria to the vaginal metabolome in eubiosis and dysbiosis a 151 panel of lactobacilli and BV associated isolates was assembled. Whole genome sequencing of seven G. vaginalis 152 strains included reference strains from the NCTC and new isolates from vaginal swabs, enables them to be assigned 153 to Clades $^{9,16,33}$ or subgroup ${ }^{34}$ and identifies genes for sialidase and vaginolysin (Table 1). Though expression was 154 not tested, all isolates carry the genes coding for sialidase and vaginolysin. Strains KC1 and KC2 have Type 1B vaginolysin while the remainder have Type $1 \mathrm{~A} .{ }^{35}$ Six of the seven strains are members of Clade $1 /$ Subgroup $\mathrm{C} / \mathrm{Clade}$ GV2a, corresponding to sequence variant G2 strains which have been shown to drive observed associations with PTB. ${ }^{9}$ The remaining isolate, $\mathrm{KC} 1$, is a member of Clade 3/Subgroup D/Clade GV1b. Tested for susceptibility to the main antibiotics used for BV, two isolates, $\mathrm{KC1}$ and $\mathrm{KC} 3$, are found to be resistant to metronidazole and tinidazole. All isolates are sensitive to clindamycin and erythromycin.

Overview of bacterial metabolism in BHI and identification of major metabolic strategies for BV associated bacteria. Analysis of $\mathrm{BHI}$ spent culture allows comparison of the overall metabolic strategy for each of the $\mathrm{BV}$ associated bacteria but also comparison (Fig. 1) of the relative amounts of key metabolites that define the vaginal chemical environment and/or have been associated with BV and/or PTB (Fig. 2; S1). The NMR metabolomic approach clearly identifies the pyruvate and/or glucose fermentative strategies of $A$. vaginae, Pe. anaerobius and the seven $G$. vaginalis isolates. The seven $G$. vaginalis isolates can be distinguished from each other and classified according to whether they use the bifid shunt (BS) alone, producing lactate and acetate from glucose, ${ }^{36}$ or mixed acid fermentation (MAF) producing lactate and acetate but also formate and ethanol and consuming pyruvate in addition to glucose (Fig. 1A; 2A/B/E/F). G. vaginalis 10287 and 10915 are hence classified as using BS alone while the remainder all use MAF.

M. curtisii is known to be capable of using trimethylamine oxide (TMAO) as an electron donor for anaerobic respiration, producing trimethylamine (TMA). ${ }^{37} \mathrm{In} \mathrm{BHI}$ it also conducts anaerobic respiration, but the production of succinate (Fig. $1 ; 2 \mathrm{C}$ ) is suggestive of fumarate acting as the electron donor in place of TMAO which is absent. $M$. curtisii is known also to consume arginine to produce ornithine, citrulline and ammonia, ${ }^{38}$ and both it and $A$. vaginae do this also in $\mathrm{BHI}$ (Fig. 1A; S1E/T). P. bivia characteristically also produces succinate via anaerobic respiration but also ferments glucose to acetate, ${ }^{39}$ and this is observed in $\mathrm{BHI}$ alongside avid consumption of asparagine (Fig. 1A; 2B/C; S1D). P. bivia notably excretes a variety of metabolites that are not produced at the same levels or at all, and are often consumed, by the other BV associated bacteria. These include succinate and fumarate and alanine, glutamate, glycine, methionine, phenylalanine, proline, valine and uracil (Fig. $1 A ; S 1 C / H / J / L / M / N / O / P / R / S$ ).

The result of these differing metabolic strategies is, in every case, an acidification of the spent BHI culture but this is relatively modest for $M$. curtisii, Pe. anaerobius and $A$. vaginae compared with that observed for the seven $G$. vaginalis strains and $P$. bivia (Fig. 1B).

182 Considering the lactobacilli, four species are considered obligate homofermentative (L. acidophilus, L. crispatus, L. gasseri and L. iners) using the Embden-Meyerhof-Parnas (EMP) pathway to make lactate (both D-lactate and Llactate with the exception of $L$. iners that makes only L-lactate), two species are considered facultative heterofermentative making lactate (L-lactate for $L$. rhamnosus and D-lactate for $L$. jensenii) and acetate and one species, $L$. fermentum, is obligate heterofermentative producing lactate, acetate and ethanol as well as $\mathrm{CO}_{2}{ }^{40}$ The present NMR results are consistent with this with all lactobacilli producing lactate (Fig. S2A; S3A), only L. fermentum producing substantial quantities of ethanol (Fig. S2A; S3E) and only L. rhamnosus producing substantial amounts of formate (Fig. S2A; S3F). Consistent with genome sequence studies, which showed a lack of enzymes to produce acetate, ${ }^{41,42}$ L. iners, is the only Lactobacillus in this study that does not produce any acetate in BHI; acetate production by the other lactobacilli varies considerably (Fig. S2A; S3B). The lactobacilli can be further distinguished, and proline (Fig. S4B/D/J/K/M/P/R) and production of alanine, valine, isoleucine and uracil (Fig. S4K/S/T/U). 
bioRxiv preprint doi: https://doi.org/10.1101/2021.11.17.468714; this version posted November 17,2021 . The copyright holder for this preprint (which was not certified by peer review) is the author/funder, who has granted bioRxiv a license to display the preprint in perpetuity. It is made available under aCC-BY 4.0 International license.

194 Acidification of the spent culture media is likely limited by the relatively low glucose concentration in BHI but the greatest acidification is achieved by L. acidophilus (significantly more than all except L. crispatus 2 ), which also produces more lactate than any of the other strains $(p<0.05)$ (Fig. S2B; S3A).
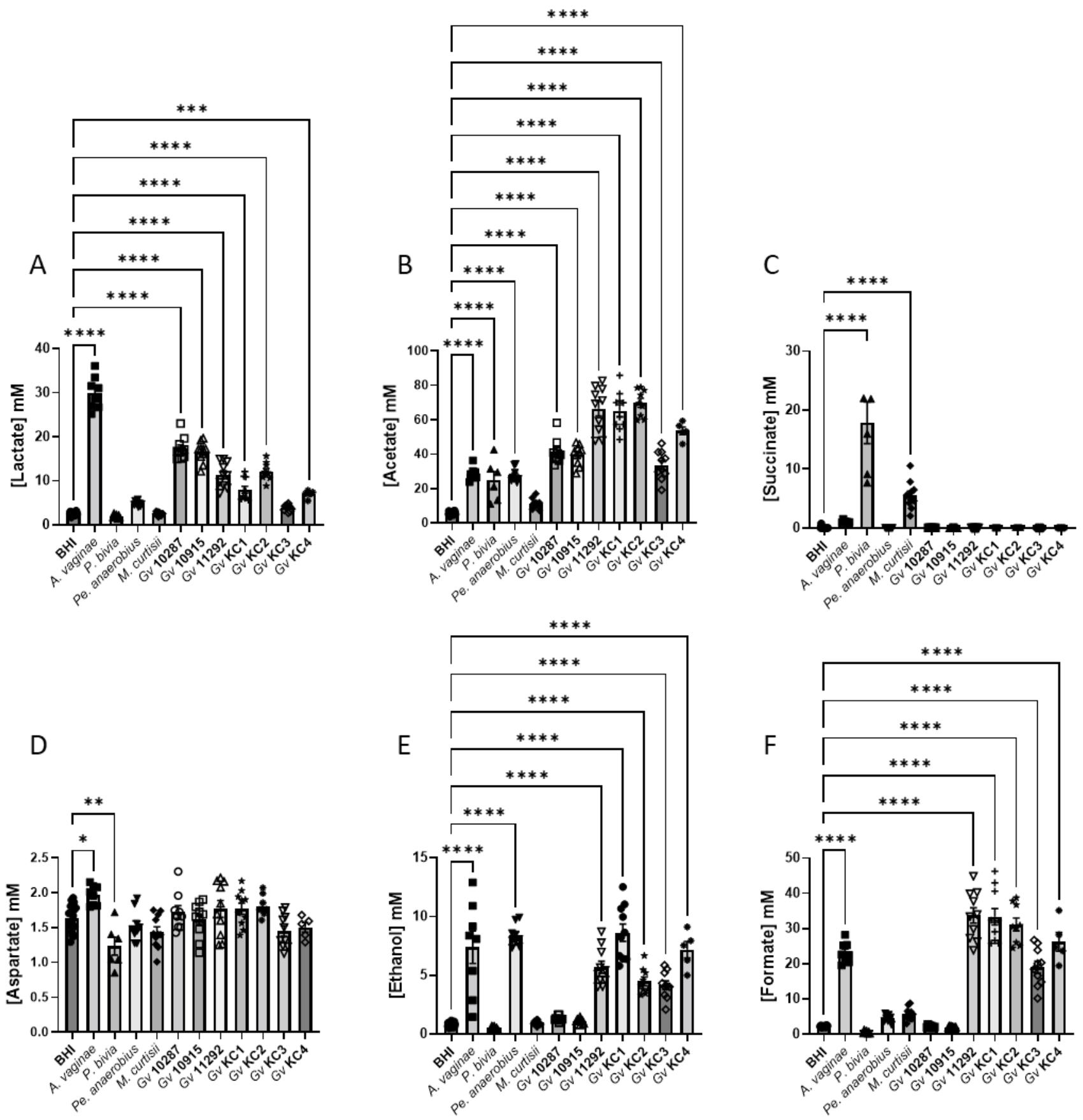

Figure 2. Production of organic acids, aspartate and ethanol by bacterial vaginosis associated bacteria in BHI. Comparisons are made between $\mathrm{BHI}$ and each spent culture as determined by One-way ANOVA with Tukey correction for multiple comparisons for the main products of fermentation and/or those involved in anaerobic respiration. Comparisons for other metabolites presented in Fig. S1. Only pairwise comparisons where $p<0.05$ are shown. ${ }^{*} p<0.05 ;{ }^{* *} p<0.01 ;{ }^{* *} p<0.001$; $^{* * * *} p$ $<0.0001$.

We have shown previously that lower lactate and higher acetate were associated with increased risk of PTB $<37$ weeks (odds ratios respectively 0.432 and 1.610$).{ }^{15}$ As expected, and again despite the relatively low concentration 

available under aCC-BY 4.0 International license.

206 culture (Fig. S3A), which substantially exceed production by BV associated bacteria (Fig. 2A). Of note however is that $A$. vaginae spent culture is enriched with around $27 \mathrm{mM}$ lactate and the two BS $G$. vaginalis produce substantially more lactate than the five MAF G. vaginalis strains $(p<0.0001)$ (Fig. $2 A)$. Except for $L$. iners, acetate is produced by lactobacilli in $\mathrm{BHI}$ to achieve final concentrations ranging from $5 \mathrm{mM}$ to $21 \mathrm{mM}$ (Fig. S3). Similar levels of acetate production are achieved by $A$. vaginae, $P$. bivia and $P$ e. anaerobius but this is dwarfed by production by $G$. vaginalis with BS strains attaining c $35 \mathrm{mM}$ and MAF strains as much as $65 \mathrm{mM}$. Although succinate secretion is a hallmark of anaerobic respiration and concentrations of nearly $18 \mathrm{mM}$ are achieved in P. bivia spent culture (Fig. 2C), small amounts of this dicarboxylate (1-4 mM) are also detected in all lactobacilli spent cultures with the exception again of $L$. iners (Fig. S3C).

Higher aspartate has previously been associated with increased risk of PTB $<37$ and $<34$ weeks (odds ratios respectively 1.675 and 1.768). ${ }^{15}$ Seven of the nine lactobacilli strains produce this, but this is very modest with spent culture enriched by a maximum of $1.2 \mathrm{mM}$ aspartate (Fig. S3D). In monoculture, none of the G. vaginalis strains produce aspartate but modest amounts are produced by $A$. vaginae and it is consumed by P. bivia (Fig. 2D). We have reported higher glucose associated with increased risk of PTB < 34 weeks (odds ratio 1.269). ${ }^{15}$ Almost all glucose in $\mathrm{BHI}$ is consumed by both lactobacilli and BV associated bacteria, with the exception of M. curtisii (Fig. $\mathrm{S} 1 \mathrm{~A} / \mathrm{S} 4 \mathrm{~A})$. G. vaginalis KC3 did not consume all glucose in this first study but subsequently it grew well, and its consumption matched that of the other $\mathrm{G}$. vaginalis strains (Fig. S1A/S7A). In contrast, pyruvate available in BHI is not universally consumed (Fig. S1B; S4B). A. vaginae, Pe. anaerobius, L. crispatus 1 and L. fermentum consume all pyruvate available while the remaining lactobacilli and BV associated bacteria, except for G. vaginalis 10287 and 10915, consume some but not all. G. vaginalis 10287 and 10915 secrete modest amounts of pyruvate into the spent culture $(p<0.05)$.

Higher leucine and betaine and lower tyrosine have also been associated with increased risk of PTB $<37$ weeks (odds rations respectively, 3.118, 1.365 and 0.023 ). ${ }^{15}$ None of the lactobacilli or BV associated bacteria in the present study produce leucine when cultured in $\mathrm{BHI}$ though it is avidly consumed by P. bivia (Fig. S1G; S4G). Tyrosine is produced in modest amounts by six of the lactobacilli isolates, most notably by L. acidophilus, L. gasseri 1 and 2 , and most G. vaginalis strains as well as $A$. vaginae, P. bivia and M. curtisii (Fig S1C). It is consumed avidly by Pe. anaerobius (Fig. S1C). With the exception of Pe. anaerobius, L. crispatus 1 and L. iners, where there is modest consumption, the concentration of betaine does not change in the spent culture of either the lactobacilli or the BV associated bacteria (Fig. S1E; S4H). Similarly, with the exception of $A$. vaginae, changes in choline concentrations are minimal (Fig S1F; S4F).

Symbiosis between P. bivia and Pe. anaerobius influences production of key PTB markers. ${ }^{1} \mathrm{H}$ NMR of the spent culture from $P$. bivia, $P$ e. anaerobius and a 1:1 co-culture reveals that combining the two species leads to a substantial adjustment in the levels of metabolites that have previously been associated with PTB and/or shown utility in predicting patient outcomes. In the spent $\mathrm{BHI}$ media, even though relative abundance could not be enumerated by plating, there is clear evidence from production and consumption of species-specific metabolites that both species proliferate (Fig. 3). In monoculture, only P. bivia consumes asparagine and produces butyrate, fumarate and succinate and this is observed also in co-culture although succinate production is reduced $(p<0.0001)$ (Fig. S5A-D). Similarly, Pe. anaerobius is known to have a characteristic organic acid production profile, ${ }^{43}$ and in monoculture, of the two species, only Pe. anaerobius consumes phenylalanine, proline, tyrosine, uracil, lysine, methionine, choline and leucine (Fig. S5J-Q) and produces ethanol, 4-methylpentanoate (isocaproate), 3-(4-hydroxyphenyl)propanoate (desaminotyrosine/phloretic acid), 3-phenylpropionate (hydrocinnamate) and 5-aminopentanoate (aminovalerate) (Fig. S5E-I). With the possible exception of choline consumption, this is also observed in coculture, with increased production observed for all five of its specific products. 

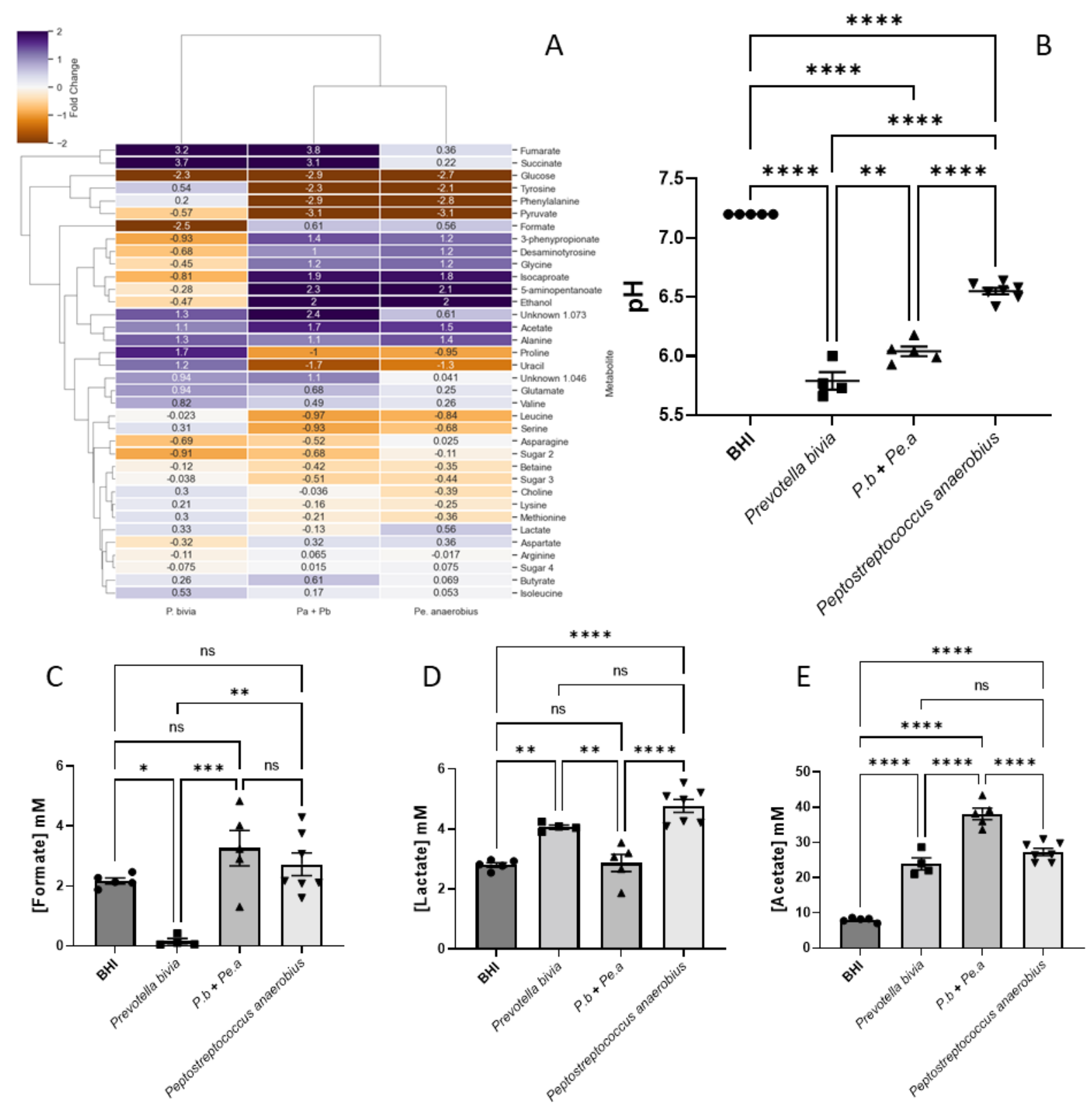

Figure 3. Commensal symbiosis of $P$. bivia and Pe. anaerobius in $\mathrm{BHI}$ generates a distinct chemical environment. The heatmap compares the metabolite fold change from ${ }^{1} \mathrm{H}$ NMR of spent culture media and enables, the major metabolites produced by each isolate and the key differences in metabolic strategy to be revealed (A). The $\mathrm{pH}$ of the three spent cultures is compared with fresh BHI (B). Levels of formate (C), lactate (D) and acetate (E) in spent culture are shown relative to fresh BHI. Further metabolites are shown in Fig. S5 and S6. Comparisons are shown between all conditions, as determined by Oneway ANOVA with Tukey correction for multiple comparisons. ${ }^{*} p<0.05,{ }^{* *} p<0.01,{ }^{* * *} p<0.001,{ }^{* * * *} p<0.0001$.

Previously, a commensal symbiosis between $P$. bivia and $P$ e. anaerobius has been demonstrated and ascribed to use of amino acids, by Pe. anaerobius, that were secreted by P. bivia. ${ }^{24}$ Here, ${ }^{1} \mathrm{H}$ NMR identifies enrichment of BHI media with tyrosine, phenylalanine, proline, methionine, alanine, glutamate, glycine, isoleucine, valine and also choline and uracil (Fig. S5/S6). Of these, Pe. anaerobius avidly consumes tyrosine, phenylalanine, proline and uracil, and modestly consumes methionine and possibly choline (Fig. S5). Levels of alanine, glutamate, glycine, isoleucine and valine are also lower in the co-culture spent media than that of $P$. bivia but, since these are available in BHI normally and are not consumed in Pe. anaerobius monoculture, it is assumed that this reduction can also be accounted for by a lower overall growth of $P$. bivia in the combination relative to monoculture (Fig. S6). 
While the benefits of co-culture to Pe. anaerobius appear manifold, the reverse is not true for $P$. bivia and ${ }^{1} \mathrm{H} N M R$ does not detect any metabolites produced by Pe. anaerobius that are consumed by $P$. bivia. This supports the previous finding of a commensal relationship between the two organisms. ${ }^{24}$ There is one possible caveat to this in that, while no effect of $P$ e. anaerobius conditioned media on $P$. bivia growth was observed previously, ${ }^{24}$ here we find that $P$. bivia metabolism is likely altered by co-culture with Pe. anaerobius. First, while production of $P e$. anaerobius specific metabolites is increased in co-culture relative to monoculture, the same is true for $P$. bivia only for butyrate ( $p=0.0295)$, with less succinate, alanine, glutamate, glycine and valine than might be expected. Second, the total consumption of formate by P. bivia in monoculture is not observed in co-culture (Fig. 3C) while lactate, produced by both species in monoculture, is no more abundant in the co-culture spent media than in fresh BHI (Fig. 3D) even though acetate production almost doubles (Fig. 3E). Both formate and lactate are potential electron donors for anaerobic respiration and the NMR analysis provides evidence for a switch in electron donor, from formate to lactate, by P. bivia when Pe. anaerobius is present.

The spent culture media $\mathrm{pH}$ will be affected by the production/consumption of a range of organic and amino acids. Although acidification of spent culture will be limited due to the relatively low levels of glucose interactions between these two species will affect the acidity of the environment (Fig. 3B). Despite production of acetate (pKa 4.76) and lactate (pKa 3.86), acidification by Pe. anaerobius is relatively modest with a reduction by only $0.65 \mathrm{pH}$ units. In contrast, both the spent $P$. bivia monoculture and co-culture are reduced by over one $\mathrm{pH}$ unit (respectively 1.41 and 1.16). In both cases substantial amounts of succinate (pKa 4.2, 5.6) are produced (20 mM vs $9.5 \mathrm{mM}$ for monoculture vs co-culture). More acetate is produced in the co-culture (30.1 vs $15.9 \mathrm{mM}$ ) but there is no net lactate production. These effects combine to ensure that the spent co-culture $\mathrm{pH}$ is a little higher than that of the $P$. bivia monoculture but substantially lower than that corresponding to Pe. anaerobius.

Symbiosis between P. bivia and G. vaginalis is strain and metabolic strategy dependent - Five G. vaginalis strains (10287, 10915, 11292, KC1 and KC3), representing both BS and MAF strategies, were selected for co-culture experiments with P. bivia NCTC 11156. With the exception of KC1, the only strain in the present study not of sequence variant $\mathrm{G} 2,{ }^{9}$ positive correlations were detected between the number of CFU identified for either species when plated following co-culture in BHI (Fig. 4B, Table 2), with the strongest positive relationship found for G. vaginalis 10287 , one of the BS strains.

The Spearman and Pearson $\mathrm{r}$ for KC1 are both negative indicating that when $G$. vaginalis KC1 grew well, P. bivia did not, and vice versa. This is manifested in the metabolomics analysis where levels of some metabolites, known to be produced by $P$. bivia, notably succinate, fumarate, proline, uracil and alanine are highly variable (Fig. S7D-F, J, K). There is some explanation for this phenomenon in the metabolomics data (Fig.4A). Notably, KC1 may be the only one of the five $G$. vaginalis strains that is not capable of adequately supplying asparagine to $P$. bivia (Fig. 4D). As noted above, $P$. bivia avidly consumes asparagine since this can be used to produce aspartate and, in turn, fumarate which is an important electron acceptor anaerobic respiration. Asparagine is produced in substantial amounts by all $G$. vaginalis isolates $(p<0.0001)$, with the exception of KC1 ( $p=0.0333)$. The two BS strains increase the availability of asparagine by $42 \%$ (10915) and $45 \%$ (10287). This is modest when compared with MAF strains 11292 and KC3 which respectively increase the availability of asparagine by $63 \%$ and $70 \%$, such that approximately double the amount of asparagine that is consumed by P. bivia in monoculture is available in co-culture. In contrast, KC1 only increases the amount available by $18.5 \%$. 
bioRxiv preprint doi: https://doi.org/10.1101/2021.11.17.468714; this version posted November 17,2021 . The copyright holder for this preprint (which was not certified by peer review) is the author/funder, who has granted bioRxiv a license to display the preprint in perpetuity. It is made available under aCC-BY 4.0 International license.
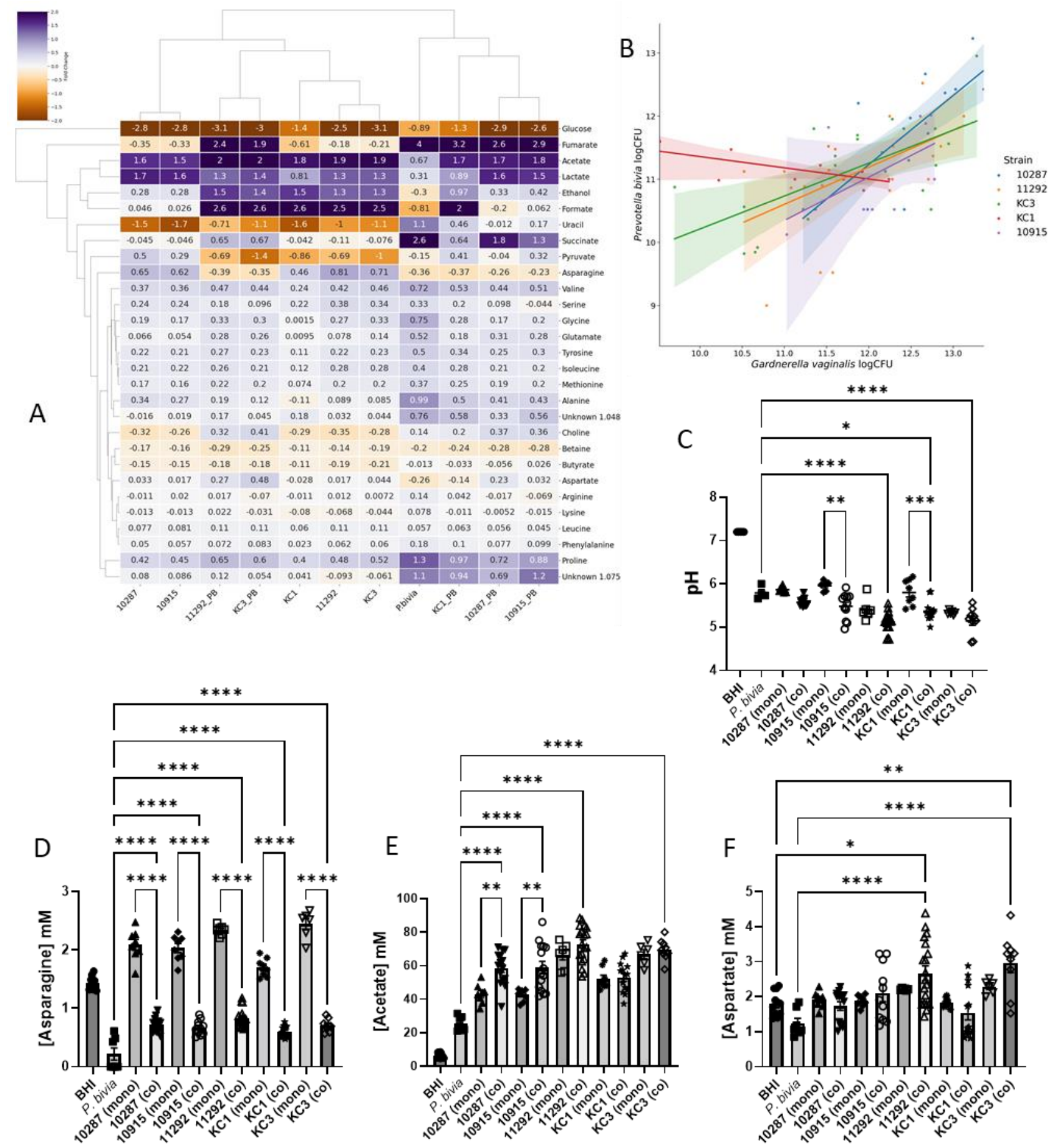

Figure 4. Co-culture of Prevotella bivia NCTC 11156 and a panel of Gardnerella vaginalis isolates. A heatmap shows the relationship between metabolite fold-changes detected by ${ }^{1} \mathrm{H}$ NMR of spent cultures (A). The correlation between $\mathrm{CFU}$ for $P$. bivia and each G. vaginalis partner is shown for five co-cultures (B); Spearman and Pearson $r$ are provided in Table 1. Spent culture $\mathrm{pH}$ for mono- and co-cultures as well as fresh $\mathrm{BHI}$ (C). G. vaginalis supplies $P$. bivia with asparagine (D). Acetate levels increase when bifid shunt $G$. vaginalis (10287 and 10915) are co-cultured with P. bivia (E). Symbiosis between $P$. bivia and MAF $G$. vaginalis strains produces aspartate $(F)$. Comparisons are shown between each co-culture and the corresponding mono-cultures (C-E) and also fresh BHI (F) as determined by One-way ANOVA with Tukey correction for multiple comparisons. Only $p<0.05$ shown; ${ }^{*} p<0.05,{ }^{* *} p<0.01,{ }^{* * *} p<0.001,{ }^{* * * *} p<0.0001$. Comparisons for further metabolites shown in Fig. S7. 
313 While supply of asparagine from $G$. vaginalis to $P$. bivia is observed for both MAF and BS strains, a further means

314 by which BS strains, but not MAF strains, may supply $P$. bivia is also apparent. Unlike the BS G. vaginalis strains, $P$. 315 bivia and all three MAF G. vaginalis strains consume pyruvate from BHI (Fig. S7C). With two species growing to316 gether the metabolite data for co-culture has greater variance but considering just the data from monocultures 317 (as above) indicates that some pyruvate is likely secreted from $10287(p=0.0035)$ and $10915(p=0.0046)$. As such 318 the BS strains differ from the MAF strains in that they avoid competition with $P$. bivia for pyruvate and, likely, may 319 supply it in co-culture.

320 As noted above, in monoculture the MAF strains 11292 and KC3 $(p<0.0001)$ and KC1 $(p<0.05)$ produce more 321 acetate than the BS strains 10287 and 10915 but less lactate. In co-culture however acetate produced by $P$. 322 bivia/10287 and P. bivia/10915 increases by $42-45 \%$ over that produced by $G$. vaginalis alone while the corresponding figure for the MAF strains is between 2 and $11 \%$. Lactate production is largely unchanged in co-culture for any of the strains. Co-culture with $P$. bivia therefore has the potential to substantially increase overall acetate levels and change the acetate/lactate ratio when BS strains are present but not MAF strains. Further, while $P$. bivia was confirmed to consume formate, ethanol and aspartate by spiking experiments (Fig. S8) there is insufficient evidence here that production of these metabolites by MAF G. vaginalis provides substantial benefit for $P$. bivia with no apparent consumption of these metabolites in the respective co-cultures (Fig. S7G, H; Fig. 4F). Indeed, while both $11292(p=0.015)$ and KC3 $(p=0.008)$ produce aspartate in monoculture, the amount found in the spent co-culture media is increased respectively 2 - and 3-fold (Fig. 4F). As previous work has indicated $P$. bivia supplies ammonia to $G$. vaginalis, ${ }^{25}$ this suggests that MAF $G$. vaginalis might be performing a detoxification role by consuming both ammonia and fumarate (Fig. S7E), secreted by P. bivia, to produce aspartate. ${ }^{44}$

While the symbiotic relationship between $P$. bivia and Pe. anaerobius is commensal in $\mathrm{BHI}$, we suggest here that the relationship between P. bivia and $G$. vaginalis is mutualistic since, as well as the presumed supply of ammonia and fumarate, we show P. bivia also likely supplies $G$. vaginalis with uracil (Fig. 5; S7J). As above, in monoculture $P$. bivia produces uracil and all five $G$. vaginalis strains consume it. Not all uracil is consumed however and in coculture the overall levels remaining in spent culture are intermediate between that obtained from $P$. bivia monoculture and available in fresh BHI. Nevertheless, pending further investigation, there is no reason to assume uracil liberated by $P$. bivia is not then available to $G$. vaginalis.

The levels of other metabolites vary little between the spent monoculture and co-cultures though choline, produced by $P$. bivia but not G. vaginalis in monoculture, is further increased in three out of the five spent co-cultures 10915, 11292, KC3; Fig S7N).

\section{Discussion}

344 The present study describes the metabolic strategies, and quantifies the relative metabolites produced and consumed, in BHI by both a panel of lactobacilli and a range of BV and/or PTB associated bacteria. Further, we characterise the effect, on metabolite consumption and excretion and consequently the likely vaginal chemical environment, of commensal symbiosis between $P$. bivia and $P$. anaerobius and a mutualistic symbiosis between $P$. bivia and $G$. vaginalis, providing mechanistic details for both. We demonstrate substantial differences in metabolite consumption/production between different strains of G. vaginalis that adopt either BS alone or MAF strategies and how this affects outcome of the mutualistic symbiosis with P. bivia. Below we consider the effects of the two symbiotic relationships before assessing how variation in metabolic strategy in lactobacilli, BV/PTB associated bacteria and symbiosis affects the vaginal chemical environment and how this may have functional impact and modify metabolite-based approaches to PTB risk prediction. 


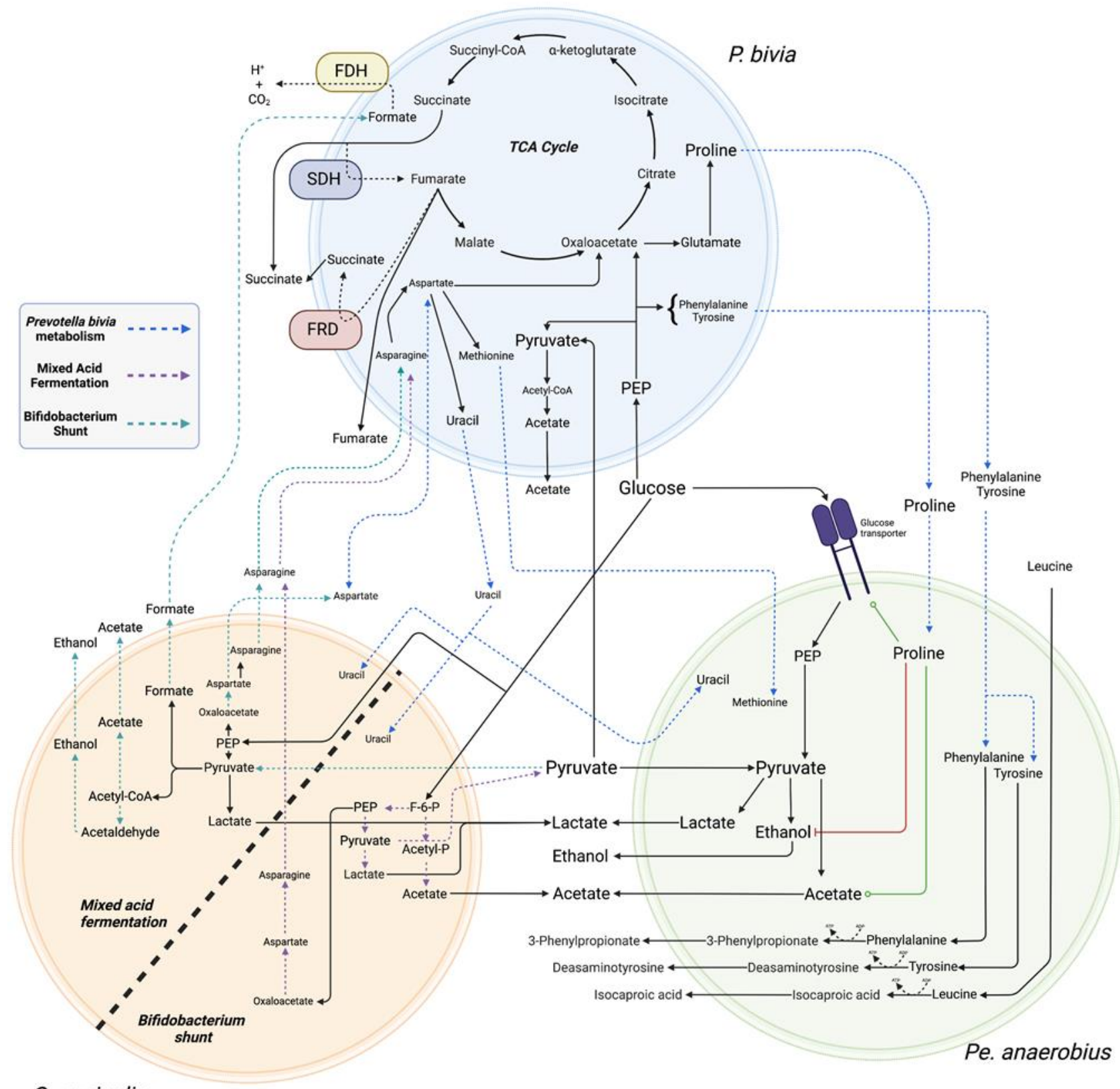

Figure 5. Commensal relationship of Prevotella bivia NCTC 11156 with Pe. anaerobius NCTC 11460 and mutualistic relationships with BS and MAF Gardnerella vaginalis. P. bivia supplies uracil, methionine, tyrosine, phenylalanine and proline to Pe. anaerobius. These all stimulate glucose uptake by Pe. anaerobius and increased proline availability also causes a switch from ethanol to acetate production, accounting for a $56 \%$ increase in acetate secretion. The relationship between $G$. vaginalis and $P$. bivia is mutualistic with the former supplying asparagine and the latter again supplying uracil. However, the relationship between MAF or BS $G$. vaginalis strains and P. bivia will differ with MAF strains competing with $P$. bivia for pyruvate but potentially supplying formate as an electron donor for anaerobic respiration. The origin of the increased aspartate found in MAF G. vaginalis and P. bivia co-culture is as yet unclear. FDH - formate dehydrogenase; SDH - succinate dehydrogenase; FRD - fumarate reductase. Image created with BioRender.com.

Commensal supply of proline by P. bivia increases acetogenesis by Pe. anaerobius - commensal symbiosis of $P$. bivia and Pe. anaerobius is known to depend on provision of amino acids from the former to the latter. ${ }^{24}$ Here we show that, in addition, uracil supply is substantial and that these amino acids are limited to methionine, tyrosine, phenylalanine and proline with the last three consumed avidly by Pe. anaerobius. All four of these amino acids have been shown to stimulate glucose uptake, with leucine and tyrosine having the greatest effect. ${ }^{45}$ The increased 
369 availability of tyrosine and phenylalanine is associated with, respectably, a $23 \%$ increase in desaminotyrosine and a 33\% increase in 3-phenylpropionate production in co-culture compared with Pe. anaerobius conditioned media. In contrast, proline availability increases by $470 \%$ in $P$. bivia conditioned media, and this leads to a $243 \%$ increase in 5-aminopentanoate production in co-culture. Proline has been shown to not only be capable of initiating glucose uptake, but also high proline levels are associated with a switch from ethanol to acetate production, a process which generates additional ATP. ${ }^{45} \mathrm{Here}$, acetate in co-culture increases by $56 \%$ over the Pe. anaerobius monoculture while the corresponding increase for ethanol is only $17 \%$. As such then, co-existence of $P$. bivia with Pe. anaerobius and/or greater availability of proline from other sources can be expected to substantially increase production of acetate (Fig. 5).

Diversity in G. vaginalis metabolism influences symbiosis with P. bivia. When originally described, G. vaginalis was proposed to be the sole aetiological agent of BV since it was found in 127 out of 138 cases but in none of 78 healthy women examined. ${ }^{27,46}$ Since then more doubt has been expressed that $G$. vaginalis alone is the causative agent of $\mathrm{BV}$ as its distribution is more widespread and is frequently found colonising the vagina of healthy or nonsymptomatic women. At the same time, there is recognition that there is considerable diversity in the G. vaginalis genus with both different species and clades or sub-groups being proposed. ${ }^{33,34,47}$ The functional relevance of diversity in $G$. vaginalis has been highlighted by the finding in one study that an association, between $G$. vaginalis and PTB, was driven exclusively by sequence variants $\mathrm{G} 2$ with an association absent for other variants and the association for the genus lost when G2 variants were excluded. ${ }^{9}$ The implication from this is that associations and mechanistic links between $G$. vaginalis and PTB, if they exist, will be obscured if diversity is not considered.

It has been shown recently that $G$. vaginalis enhances the invasive potential of $P$. bivia ${ }^{26}$ aiding its ascension into the uterus. A commensal metabolic symbiotic relationship between these two species was proposed over 20 years ago. ${ }^{25}$ Here we use NMR metabolomics to characterise the symbiosis between P. bivia and G. vaginalis. Of the five $G$. vaginalis isolates tested here, those four that are identified as sequence variants $\mathrm{G} 2,{ }^{9}$ benefit from a relationship that this is mutualistic rather than commensal and further show that the outcome is specific to the metabolic strategy of specific $G$. vaginalis isolates. As such we show that diversity in $G$. vaginalis metabolism is manifested both in monoculture and co-culture and has potential to alter the vaginal chemical environment. Lower lactate and higher acetate levels in the vagina are considered hallmarks of BV and are associated with sPTB. ${ }^{15,17,22}$ Such conditions would be consistent with a depletion of lactobacilli and increase in $G$. vaginalis, but this relative difference also describes the relationship between BS and MAF strains of $G$. vaginalis albeit not to the same magnitude. Further, since co-culture of MAF strains, but not BS strains, with $P$. bivia leads to an increase in aspartate production, this diversity impacts on an important metabolite predictor of SPTB. ${ }^{15}$

Functional impact and implications for risk prediction of BV associated bacteria and lactobaccili metabolism. Low vaginal $\mathrm{pH}$ and high lactate are both associated with protective benefits while short chain fatty acids (SCFAs) including acetate, butyrate and succinate (and propionate where present) have pleiotropic effects in inflammation. ${ }^{18-21} \mathrm{~A}$ recent comparison of the effects of treating cervicovaginal epithelial cells with mixtures of organic acids representing optimal (33 $\mathrm{mM}$ lactic acid/lactate, $4 \mathrm{mM}$ acetic acid, $1 \mathrm{mM}$ succinic, butyric and propionic acids) and non-optimal ( $6 \mathrm{mM}$ lactate, $100 \mathrm{mM}$ acetate, $20 \mathrm{mM}$ succinate and $4 \mathrm{mM}$ butyrate and propionate) vaginal microbiota, respectively at $\mathrm{pH} 3.9$ and $\mathrm{pH} \mathrm{7,} \mathrm{revealed} \mathrm{that} \mathrm{the} \mathrm{mixture} \mathrm{chosen} \mathrm{to} \mathrm{mimic} \mathrm{BV} \mathrm{increased} \mathrm{basal} \mathrm{and}$ toll-like receptor (TLR) induced production of pro-inflammatory cytokines including tumor necrosis factor- $\alpha$ (TNFa) but decreased basal production of CCL5 and IP-10 chemokines. ${ }^{20}$ When tested alone, $100 \mathrm{mM}$ acetate at pH 7 largely recapitulated the effects of the BV mixture. Since the pKa of acetic acid is 4.75 and those of succinic acid are 4.2 and 5.6, these will exist respectively as the carboxylate or dicarboxylate anions at such an extreme as $\mathrm{pH}$ 7. As both the relative concentrations and the ionization state of the organic acids are changing under these experimental conditions, it is yet unclear as to the relative importance of these two factors and the impact of acetic 
413 acid/acetate may depend also on the vaginal $\mathrm{pH}$, driven by relative concentrations of, primarily lactic acid. The

414 absolute and relative proportions of these two organic acids may therefore have substantial impact on the vaginal 415 inflammatory state and need to be considered.

416 The description of metabolism, in pairings of $P$. bivia with diverse $G$. vaginalis isolates, reveals symbiosis has the 417 potential to substantially increase the amount of acetate excreted by BS but not MAF strains. Similarly, co-culture 418 between $P$. bivia and $P$ e. anaerobius modulates $\mathrm{pH}$, eliminates net lactate production and increases acetate pro419 duction. Together, these observations raise the prospect that co-existence of $P$. bivia with either of the two species 420 might affect their physiological impact.

421 Further, while this study is predominantly focused on the metabolism of PTB and/or BV associated bacteria, it is 422 also important to consider the metabolism of lactobacilli that often dominate the vaginal microbiome, and hence 423 contribute to the metabolite background, and their known relationships with BV/PTB associated bacteria. Patterns 424 of cooccurrence between L. crispatus and G. vaginalis have been shown to be highly exclusive. ${ }^{9}$ In contrast, L. iners 425 has been shown to co-exist with $G$. vaginalis at high frequency and its dominance has been found to be associated with preterm birth ${ }^{10}$. Since this work confirms $L$. iners is incapable of making acetate, ${ }^{41,42}$ all acetate detected in an $L$. iners dominated sample will originate from other bacteria, frequently $G$. vaginalis, and the change in acetate levels may be expected to be larger in such situations than observed where other lactobacilli dominate or that are considered mixed dysbiotic. This may have implications both for inflammation and risk prediction. Indeed, acetate production by lesser producers (A. vaginae, P. bivia and perhaps BS G. vaginalis) may be easier to detect in low acetate background as found in L. iners dominated CST compared with other backgrounds, i.e. L. crispatus or where other lactobacilli co-exist e.g. L. rhamnosus, and the relative change will be greater. Similarly, although less abundant, succinate is produced by nine out of eleven lactobacilli strains tested here, with none detected for $L$. iners and L. rhamnosus 1. Again, detection of succinate produced by PTB and/or BV associated P. bivia and BV associated $M$. curtisii will be easier to detect in the L. iners CST background than in others.

A comparison between representative isolates of $L$. iners and $L$. crispatus dominated microbiomes is therefore warranted but beyond the scope of the present study. Notably, substantial variation in metabolism was observed in the two L. crispatus isolates, notably for asparagine consumption and aspartate and acetate production, and there is a need to establish the extent to which metabolism varies across a larger panel of isolates to appreciate its possible impact.

Finally, we assess whether the current study sheds any light on the protection against PTB suggested to be provided by L. acidophilus. ${ }^{15}$ Of note $L$. acidophilus does make the highest amount of lactate of all the lactobacilli isolates grown here in $\mathrm{BHI}(p<0.0001$ for all but L. rhamnosus $p<0.05$ and $L$. jensenii $2 p=0.0047)$ and it produces the spent culture with the lowest $\mathrm{pH}$. Lactate production is correlated with $\mathrm{H}_{2} \mathrm{O}_{2}$, which would inhibit anaerobes, and bacteriocins lose activity and hydrogen peroxide becomes unstable as the $\mathrm{pH}$ increases. Peroxide is however only produced in presence of oxygen and L. gasseri may make more $\mathrm{H}_{2} \mathrm{O}_{2}$ while cervicovaginal fluid has been shown to attenuate its microbicidal activity. ${ }^{48,49}$ As such, the extent to which higher lactate production and greater ability to acidify the environment, from certain less-dominant lactobacilli, is protective against BV or PTB should be explored further, especially if able to co-exist within more diverse communities.

\section{Conclusion}

451 The diversity of intraspecies BV/PTB associated bacteria, and interspecies lactobacilli, metabolism as well as the 452 commensal and mutualistic symbiotic relationships of $P$. bivia have the potential to alter pro-inflammatory ace453 tate, and other metabolites in the vaginal metabolome and consequently alter risk of bacterial vaginosis and/or 454 spontaneous preterm birth. 
ASSOCIATED CONTENT

Supporting Information. Further comparison of metabolites produced by BV associated bacteria, lactobacilli and the effect of co-culture is provided as Supplementary Figures S1-S8.

\section{AUTHOR INFORMATION}

\section{Corresponding Author}

460 * A. James Mason, King's College London, Institute of Pharmaceutical Science, Franklin-Wilkins Building 150 Stam461 ford Street, London, SE1 9NH, UK; Tel: + 44207848 4813; Fax: + 44207848 4800; E-mail: james.mason@kcl.ac.uk

\section{Author Contributions}

$463 \mathrm{VH}, \mathrm{CKH}, \mathrm{RMT}, \mathrm{JMS}$ and AJM designed the study. VH and AJM wrote the main manuscript text and prepared all 464 figures. Assisted by JC, JH and GH, VH conducted all bacterial culture and NMR metabolomics experiments and, 465 together with AJM, analysed the data. MEW carried out the analysis of whole genome sequence data. VA and CKH 466 obtained isolates from swabs supplied by RMT. All authors approved the manuscript.

\section{$467 \quad$ Notes}

468 The authors declare no competing interests.

469 Acknowledgment: NMR experiments described in this paper were carried out using the facilities of the Centre for 470 Biomolecular Spectroscopy, King's College London using instruments acquired with a Multi-user Equipment Grant 471 from the Wellcome Trust and an Infrastructure Grant from the British Heart Foundation. We thank Dr Andrew 472 Atkinson, Dr Adrien Le Guennec and Dr James Jarvis for assistance with liquid-state NMR experiments performed 473 at KCL. VH was supported by a King's College London iCASE award, affiliated to the London Interdisciplinary Doc474 toral Programme (LIDo), and Public Health England. Funding for the INSGHT cohort providing swabs was provided 475 from Tommy's Charity (no. 1060508); NIHR Biomedical Research Centre (BRC) based at Guy's and St. Thomas' 476 National Health Service Foundation Trust, and the Rosetrees Trust (charity no. 298582) (M303-CD1). The views 477 expressed are those of the author(s) and not necessarily those of the NHS, the NIHR, or the Department of Health 478 and Social Care. We thank Collette Allen at SDH for providing patient swabs. 
bioRxiv preprint doi: https://doi.org/10.1101/2021.11.17.468714; this version posted November 17,2021 . The copyright holder for this preprint (which was not certified by peer review) is the author/funder, who has granted bioRxiv a license to display the preprint in perpetuity. It is made available under aCC-BY 4.0 International license.

\section{REFERENCES}

480 1. Onderdonk, A.B., Delaney, M.L. \& Fichorova, R.N. The human microbiome during bacterial vaginosis. Clin. Microbiol. Rev. 29, 223-238 
bioRxiv preprint doi: https://doi.org/10.1101/2021.11.17.468714; this version posted November 17,2021 . The copyright holder for this preprint (which was not certified by peer review) is the author/funder, who has granted bioRxiv a license to display the preprint in perpetuity. It is made available under aCC-BY 4.0 International license.

30. Dieterle, F., Ross, A., Schlotterbeck, G. \& Senn, H. Probabilistic quotient normalization as robust method to account for dilution of complex biological mixtures. Application in ${ }^{1} \mathrm{H}$ NMR metabonomics. Anal. Chem. 78, 4281-4290 (2006).

31. Bolger, A.M., Lohse, M. \& Usadel, B. Trimmomatic: a flexible trimmer for Illumina sequence data. Bioinformatics 30, 2114-2120 (2014).

32. Bankevich, A. et al. SPAdes: a new genome assembly algorithm and its applications to single-cell sequencing. J Comput. Biol. 19, 455-477 (2012).

33. Ahmed, A. et al. Comparative Genomic Analyses of 17 Clinical isolates of Gardnerella vaginalis provide evidence of multiple genetically isolated clades consistent with subspeciation into genovars. J. Bacteriol. 194, 3922-3937 (2012).

34. Schellenberg, J.J., Jayaprakash, T.P., Gamage, N.W., Patterson, M.H., Vaneechoutte, M. \& Hill, J.E. Gardnerella vaginalis subgroups defined by cpn60 sequencing and sialidase activity in isolates from Canada, Belgium and Kenya. PLoS ONE 11, e0146510 (2016).

35. Garcia, E.M., Serrano, M.G., Edupuganti, L. Edwards, D.J., Buck, G.A. \& Jefferson, K.K. Sequence comparison of vaginolysin from different Gardnerella species. Pathogens 10, 86 (2021).

36. Gavini, F., Van Esbroeck, M., Touzel, J.P., Fourment, A. \& Goossens, H. Detection of fructose-6-phosphate phosphoketolase (F6PPK), a key enzyme of the Bifid-Shunt, in Gardnerella vaginalis. Anaerobe 2, 191-193 (1996).

37. Cruden, D.L. \& Galask, R.P. Reduction of trimethylamine oxide to trimethylamine by Mobiluncus strains isolated from patients with bacterial vaginosis. Microobial Ecol. Health Dis. 1, 95-100 (1988).

38. Spiegel, C.A. \& Roberts, M. Mobiluncus gen. nov. Mobiluncus curtisii subsp. curtisii sp. nov. Mobiluncus curtisii subsp. holmesii subsp. nov., and Mobiluncus mulieris sp. nov., curved rods from the human vagina. Int. J. Systematic Bacteriol. 34, 177-184 (1984).

39. Holdeman, L.V. \& Johnson, J.L. Bacteroides disiens sp. Nov. and Bacteroides bivius sp. Nov. from human clinical infections. Int. J. Systematic. Bacteriol. 27, 337-345 (1977).

40. Ruzal, S.M. (Ed.) Lactobacillus Genomics and Metabolic Engineering. Caister Academic Press, 9781910190890 (2019).

41. Macklaim, J.M., Gloor, G.B., Anukam, K.C., Cribby, S. \& Reid, G. At the crossroads of vaginal health and disease, the genome sequence of Lactobacillus iners AB-1. Proc. Natl. Acad. Sci. USA 108, 4688-4695 (2011).

42. France, M.T., Mendes-Soares, H. \& Forney, L.J. Genomic comparisons of Lactobacillus crispatus and Lactobacillus iners reveal potential ecological drivers of community composition in the vagina. App. Environ. Microbiol. 82, 7063-7073 (2016).

43. Turgeon, D.K., Bartley, S.L. \& Dowell, V.R. Use of modified norleucine-tyrosine broth in identification of Peptostreptococcus anaerobius. J. Clin. Microbiol. 28, 2120-2121 (1990).

44. Schleicher, L., Herdan, S., Fritz, G., Trautmann, A., Seifert, J. \& Steuber, J. Central carbon metabolism, sodium-motive electron transfer, and ammonium formation by the vaginal pathogen Prevotella bivia. Int. J. Mol. Sci. 22, 11925 (2021).

45. Curtis, M.A., Wittenberger, C.L. \& Thompson, J. Proline requirement of glucose utilisation by Peptostreptococcus anaerobius ATCC 27337. Infect. Immun. 55, 352-357 (1987).

46. Gardner, J.L. \& Dukes, C.D. Haemophilus vaginalis vaginitis: a newly defined specific infection previously classified non-specific vaginitis. Am. J. Obstetr. Gynecol. 69, 962-976 (1955).

47. Vaneechoutte, M., Guschin, A., Van Simaey, L., Gansemans, Y., Van Nieuwerburgh, F., Cools, P. Emended description of Gardnerella vaginalis and description of Gardnerella leopoldii sp. nov., Gardnerella piotii sp. nov. and Gardnerella swidsinskii sp. nov., with delineation of 13 genomic species within the genus Gardnerella. Int. J. Syst. Evol. Microbiol. 69, 679-687 (2019).

48. Strus, M., Brzychczy-Włoch, M., Gosiewski, T., Kochan, P. \& Heczko, P.B. The in vitro effect of hydrogen peroxide on vaginal microbial communities. FEMS Immunol. Med. Microbiol. 48, 56-63 (2006).

49. O'Hanlon, D.E., Lanier, B.R., Moench, T.R. \& Cone, R.A. Cervicovaginal fluid and semen block the microbicidal activity of hydrogen peroxide produced by vaginal lactobacilli BMC Infect. Dis. 10, 120 (2010). 
bioRxiv preprint doi: https://doi.org/10.1101/2021.11.17.468714; this version posted November 17,2021 . The copyright holder for this preprint (which was not certified by peer review) is the author/funder, who has granted bioRxiv a license to display the preprint in perpetuity. It is made available under aCC-BY 4.0 International license.

\section{Tables}

576 Table 1. G. vaginalis strain characteristics. All strains are positive for the genes encoding sialidase and vaginolysin. Concord577 ant MICs are reported from three independently repeated experiments. ng = no growth.

\begin{tabular}{|c|c|c|c|c|c|c|c|}
\hline \multirow[b]{2}{*}{ Strain } & \multirow{2}{*}{$\begin{array}{c}\text { Clade }^{34} / \text { Sub- } \\
\text { group }{ }^{35} / \text { Sequence } \\
\text { variant }^{9}\end{array}$} & \multirow{2}{*}{$\begin{array}{l}\text { Genome } \\
\text { size (kb) }\end{array}$} & \multirow{2}{*}{$\begin{array}{c}\text { GC content } \\
\text { (\%) }\end{array}$} & \multicolumn{4}{|c|}{$\mathrm{MIC}(\mu \mathrm{g} / \mathrm{ml})$} \\
\hline & & & & Clindamycin & $\begin{array}{l}\text { Erythromy- } \\
\text { cin }\end{array}$ & $\begin{array}{l}\text { Metronida- } \\
\text { zole }\end{array}$ & Tinidazole \\
\hline NCTC 10287 & 1/C/G2 & 1663 & 41.3 & 0.015625 & 0.015625 & 4 & $2-4$ \\
\hline NCTC 10915 & $1 / \mathrm{C} / \mathrm{G} 2$ & 1665 & 41.2 & 0.03125 & 0.015625 & 4 & 2 \\
\hline NCTC 11292 & 1/C/G2 & 1659 & 41.3 & 0.03125 & 0.015625 & 4 & $2-4$ \\
\hline KC1 & 3/D/G1 & 1542 & 43.3 & 0.015625 & 0.015625 & $>256$ & 128 \\
\hline KC2 & $1 / \mathrm{C} / \mathrm{G} 2$ & 1657 & 41.3 & 0.0625 & 0.015625 & 2 & $1-8$ \\
\hline KC3 & 1/C/G2 & 1733 & 41.1 & 0.0625 & 0.03125 & 16 & $64-128$ \\
\hline KC4 & $1 / C / G 2$ & 1660 & 41.3 & ng & 0.0078125 & 4 & ng \\
\hline
\end{tabular}


bioRxiv preprint doi: https://doi.org/10.1101/2021.11.17.468714; this version posted November 17, 2021. The copyright holder for this preprint (which was not certified by peer review) is the author/funder, who has granted bioRxiv a license to display the preprint in perpetuity. It is made available under aCC-BY 4.0 International license.

579 Table 2. P. bivia vs $G$. vaginalis co-culture correlation. Relationship between CFU counts for each species as a function of $G$. 580 vaginalis isolate as determined by parametric Pearson or non-parametric Spearman correlation coefficients. Only for KC1 is a 581 negative correlation between the two species found while positive correlations exist for the remaining four isolates.

\begin{tabular}{cccccc} 
G. vaginalis strain & $\begin{array}{c}\text { Number of XY } \\
\text { pairs }\end{array}$ & \multicolumn{2}{c}{ Spearman } & \multicolumn{2}{c}{ Pearson } \\
NCTC 10287 & 16 & $\mathrm{P}$ (two-tailed) & $r$ & 0.7781 & 0.0004 \\
NCTC 10915 & 9 & 0.7917 & 0.0005 & 0.5419 & 0.1318 \\
NCTC 11292 & 22 & 0.6442 & 0.0694 & 0.5133 & 0.0146 \\
KC1 & 10 & 0.5457 & 0.0086 & -0.6896 & 0.0274 \\
KC3 & 19 & -0.4768 & 0.1645 & 0.6392 & 0.0032
\end{tabular}

